DOI: http://dx.doi.org/10.18203/2320-1770.ijrcog20172313

Original Research Article

\title{
Maternal outcome in thyroid dysfunction
}

\author{
Manju V. K., Sathiamma P. K.* \\ Department of Obstetrics and Gynecology, Government TD Medical College Alappuzha, Kerala, India
}

Received: 05 March 2017

Revised: 23 April 2017

Accepted: 01 May 2017

\section{*Correspondence:}

Dr. Sathiamma P. K.,

E-mail: sathiammapk@ymail.com

Copyright: () the author(s), publisher and licensee Medip Academy. This is an open-access article distributed under the terms of the Creative Commons Attribution Non-Commercial License, which permits unrestricted non-commercial use, distribution, and reproduction in any medium, provided the original work is properly cited.

\section{ABSTRACT}

Background: Thyroid hormone status in pregnancy is an important predictor of pregnancy outcome regarding both the mother and fetus. Objective was study the maternal outcome in antenatal women presenting with thyroid dysfunction.

Methods: This descriptive study was done in 449 pregnant ladies who attended Obstetrics and Gynecology Department Government TD Medical College, Alappuzha, Kerala, India during the period January 2014 to June 2015. Aim was studying the maternal outcome in those diagnosed to have thyroid dysfunction evidenced by abnormal thyroid function tests (TFT) done in each trimester.

Results: Majority had subclinical hypothyroidism (SCH). Common complications were preeclampsia spontaneous abortion, anemia and recurrent pregnancy loss. Infertility, post-partum hemorrhage and abruption were significantly high in overt hypothyroidism. Anemia was significantly high in subclinical hypothyroidism.

Conclusions: It is important to screen all pregnant women for thyroid dysfunction during first antenatal visit itself to facilitate early diagnosis and treatment and also can counsel regarding the adverse pregnancy outcome.

Keywords: Maternal outcome, Subclinical hypothyroidism, Thyroid dysfunction, Thyroid function tests

\section{INTRODUCTION}

Thyroid hormone status in pregnancy is an important predictor of pregnancy outcome regarding both the mother and fetus. Recent epidemiological study highlights tremendous increase in thyroid disorders in India, hypothyroidism being the most common one. Both hypothyroidism and hyperthyroidism have adverse maternal and fetal outcome if not treated adequately and timely. According to Sahu MT et al there is a wide variation in the prevalence of thyroid dysfunction with subclinical hypothyroidism found in $6.47 \%$ and overt hypothyroidism in $4.58 \%$ of pregnant women. ${ }^{1}$ Hyperthyroidism is less commonly encountered in pregnancy with a prevalence of $0.2-0.6 \%$ in study conducted by Nambiar et al both overt and subclinical dysfunctions are at high risk for complications like infertility, miscarriage, recurrent pregnancy loss, preeclampsia, preterm delivery, placental abruption, anemia and higher incidence of caesarean section if not treated timely. ${ }^{2}$

Thyroid hormonal status both production and metabolism shows changes in pregnancy. Trimester specific reference ranges in hormone values are essential and appropriate monitoring of thyroid-stimulating hormone (TSH) levels avoid adverse effects in the pregnant women and fetus. First trimester seems to be crucial where hypothyroidism can cause adverse maternal and fetal outcomes. Poorer the control, the risk will be more. Pregnant women with thyroid dysfunction should have team approach by the general practitioner, endocrinologist and obstetrician for 
better outcome. Management guidance can be offered by Endocrinologist and clinicians involved throughout the pregnancy can identify untoward effects earlier and treat accordingly.

Detection of thyroid dysfunction in pregnancy is usually incidental either by TSH screening in asymptomatic lady without previous history of thyroid disorders or by the routine checking of thyroid hormone levels in women with a previous history of thyroid disease. TSH screening just before conception in women with a known history of any thyroid disorder is recommended by most clinicians. Even if it is normal at or before conception, TSH levels should be rechecked at about four weeks of gestation in all women. Stagnaro-Green, Abalovich, et al suggest screening of patients at high risk for hypothyroidism but case-detection based on risk factors like family history of the disease, symptoms suggestive of thyroid diseases may miss a very high proportion of thyroid abnormalities in pregnant women compared with universal screening. ${ }^{3}$

Because of the high prevalence of the disease in India universal screening should be mandatory in 1st antenatal visit by measuring TSH levels and ideally screening should be carried out as soon as pregnancy is confirmed.

Objective of present work was to study the Maternal Outcome in Antenatal Women Presenting with Thyroid Dysfunction Attending Obstetrics and Gynecology Department, TDMC Alappuzha Kerala from January 2014 to June 2015.

\section{METHODS}

It is a descriptive study conducted at Government TDMC Alappuzha. Samples were selected from outpatient department, labor room, antenatal wards, and postnatal wards. Pregnant ladies with abnormal TFT (TSH, freeT3, freeT4) attending OBG department in TDMC Alappuzha during the period January 2014 to June 2015 were studied. According to American Thyroid Association guidelines, normal TSH level is- 0.1 to $2.5,0.2$ to 3 , 0.3 to3milli IU/L in first, second and 3rd trimesters respectively and these values were taken as reference for this study. In pregnancy, any value below these are hyperthyroidism and above are hypothyroidism. When T3, T4 values are normal and TSH is abnormal the disease is subclinical. Pregnant ladies detected to have thyroid disorder TFT was done in each trimester, treatment provided accordingly and any complications developing during the course of pregnancy noted. Out of the 449 -case selected for study $47(10.5 \%)$ cases had spontaneous miscarriage in 1 st trimester. The remaining 402 cases followed up till delivery. Data analyzed and expressed in its frequency and percentage.

\section{RESULTS}

During the study period, there were 6762 deliveries. Out of the $449(6.2 \%)$ women having various types of thyroid disorders 402 cases continued pregnancy till delivery and rest were miscarriage. Among the 449 women studied we found $324(72.2 \%)$ had $\mathrm{SCH}$, overt hypothyroidism for $93(20.7 \%)$ cases, $6(1.3 \%)$ women had subclinical hyperthyroidism and overt hyperthyroidism for 24 $(5.3 \%)$. Thyroid nodule and thyroid cancer noted for 2 $(0.4 \%) 1$ thyroid crises $(0.2 \%)$ and none of the cases developed postpartum thyroiditis (Table 1). Significant percentage were between the age 20-25 year, total 198 women (44.1\%) next being between 26-30 yrs. Majority were from rural area, belonging to middle socioeconomic group Almost all were booked cases.

Table 1: Types of thyroid dysfunction.

\begin{tabular}{|lll|}
\hline Types of thyroid dysfunction & Frequency & $\%$ \\
\hline Subclinical hypothyroidism & 324 & 72 \\
\hline Sub clinical hyperthyroidism & 6 & 1.37 \\
\hline Overt hypothyroidism & 93 & 20.7 \\
\hline Overt hyperthyroidism & 24 & 5.3 \\
\hline Thyroid nodule and cancer & 2 & 0.4 \\
\hline Postpartum thyroiditis & 0 & \\
\hline
\end{tabular}

SCH was more in $20-25$ years $(81.8 \%)$. Out of 13 women $>35 y$ rs overt hypothyroidism was more $(61.5 \%)$ and other type of thyroid dysfunction was more in below 20 years age group (Table 2) Majority were Primi gravida 262 cases $(58.4 \%)$, Gravida-2 was $113(25.1 \%)$ and >G3 was $74(16.4 \%)$ cases (Table 3).

Table 2: Comparison of thyroid dysfunction based on age.

\begin{tabular}{|llllllll|}
\hline SCH & & \multicolumn{5}{c}{$\begin{array}{l}\text { Overt } \\
\text { hypothyroid }\end{array}$} & Others \\
\hline Age & Cases & $\%$ & Cases & $\%$ & Cases & $\%$ \\
\hline$<20$ & 16 & 76.2 & 3 & 14.3 & 2 & 9.5 \\
\hline $20-25$ & 162 & 81.8 & 24 & 12.1 & 12 & 6.1 \\
\hline $26-30$ & 103 & 69.1 & 34 & 22.8 & 12 & 8.1 \\
\hline $31-35$ & 39 & 57.4 & 24 & 35.3 & 5 & 7.4 \\
\hline$>35$ & 4 & 30.8 & 8 & 61.5 & 1 & 7.7 \\
\hline
\end{tabular}

Table 3: Comparison of thyroid dysfunction based on age.

\begin{tabular}{|llllllll|}
\hline SCH & & \multicolumn{5}{c}{$\begin{array}{l}\text { Overt } \\
\text { hypothyroid }\end{array}$} & Others \\
\hline $\begin{array}{l}\text { OBS } \\
\text { score }\end{array}$ & Count & $\%$ & Count & $\%$ & Count & $\%$ \\
\hline PRIMI & 203 & 77.5 & 40 & 15.3 & 19 & 7.3 \\
\hline G2 & 84 & 74.3 & 23 & 20.4 & 6 & 5.3 \\
\hline > G2 & 37 & 50 & 30 & 40.5 & 7 & 9.5 \\
\hline
\end{tabular}

$16.3 \%$ of the women had previous history of thyroid dysfunction. Since we do 1 st trimester screening for thyroid dysfunction for all women, most of the diseased were diagnosed in 1 st trimester itself, only $0.4 \%$ was left undiagnosed in first trimester. Past history of thyroid dysfunction was present in $24.1 \%$ and $0.4 \%$ not having 
thyroid dysfunction in previous pregnancy developed it in this pregnancy.

No significant complications were found in 224 (49.9\%) of women. Preeclampsia and miscarriage were the most common complications found associated with thyroid dysfunction 47 ladies each (10.5\%).

Prolonged period of infertility, recurrent abortions, abruption, post-partum hemorrhage were found significantly high in overt hypothyroidism than others. Normocytic anemia was found significantly high in cases of subclinical hypothyroidism 44 cases (9.8\%) (Table 4).
Table 4: Maternal complications.

\begin{tabular}{|lll|}
\hline Maternal complications & Frequency & $\%$ \\
\hline Nil & 224 & 49.9 \\
\hline Thyroid crisis & 1 & 0.2 \\
\hline Miscarriage & 47 & 10.5 \\
\hline Pre-eclampsia & 47 & 10.5 \\
\hline Abruption & 5 & 1.1 \\
\hline Anemia & 44 & 9.8 \\
\hline PPH & 17 & 3.8 \\
\hline Infertility & 17 & 3.8 \\
\hline Threatened abortion & 11 & 2.4 \\
\hline Recurrent fetal loss & 25 & 5.6 \\
\hline
\end{tabular}

Table 5: Comparison of maternal outcome based on thyroid dysfunction.

\begin{tabular}{|c|c|c|c|c|c|c|}
\hline \multirow[b]{2}{*}{ Maternal outcome } & \multicolumn{2}{|c|}{ Subclinical hypothyroidism } & \multicolumn{2}{|c|}{ Overt hypothyroidism } & \multicolumn{2}{|l|}{ Others } \\
\hline & Count & $\%$ & Count & $\%$ & Count & $\%$ \\
\hline Nil & 187 & 57.7 & 22 & 23.7 & 15 & 46.9 \\
\hline Spontaneous abortion & 25 & 7.7 & 9 & 9.7 & 1 & 3.1 \\
\hline Pre-eclampsia & 44 & 13.6 & 18 & 19.4 & 2 & 6.3 \\
\hline Abruption & 4 & 1.2 & 6 & 6.5 & 1 & 3.1 \\
\hline Anemia & 39 & 12 & 5 & 5.4 & 7 & 21.9 \\
\hline $\mathrm{PPH}$ & 10 & 3.1 & 6 & 6.5 & 6 & 18.8 \\
\hline Recurrent pregnancy loss & 12 & 3.7 & 24 & 25.8 & 1 & 3.1 \\
\hline Infertility & 9 & 2.8 & 15 & 16.1 & 2 & 6.3 \\
\hline Threatened abortion & 10 & 3.1 & 1 & 1.1 & 1 & 3.1 \\
\hline Thyroid crisis & 0 & 0 & 1 & 1.1 & & \\
\hline Thyrotoxicosis & 0 & 0 & 0 & 0 & 0 & \\
\hline
\end{tabular}

\section{DISCUSSION}

When pregnancy is compounded by thyroid dysfunction there is increased risk for mother and fetus. Disorders of thyroid hormone and their treatment can affect fertility, maternal well-being, fetal growth and development. Pregnancy induced physiological changes exacerbate or improve thyroid disorders. Milder forms of hypo or hyper dysfunctions will not render women infertile but still associated with miscarriage. Chan $\mathrm{S}$ et al recommended that TSH should not be considered in isolation for detection of thyroid disorders but always should be measured in conjunction with Free T4 and Free T3. ${ }^{4}$ According to James D different types of thyroid disorders in pregnancy include subclinical and overt hypothyroidism, subclinical and overt hyperthyroidism, thyroid nodule, cancer and postpartum thyroiditis. ${ }^{5}$ Among 449 women studied $72.2 \%$ had SCH, $1.3 \%$ had subclinical hyperthyroidism, overt hypothyroidism for $20.7 \%$ and overt hyperthyroidism in $5.3 \%$, thyroid nodule and malignancy for $0.4 \%$ thyroid crisis for $0.2 \%$ cases and none developed postpartum thyroiditis. In a study by Ajmani et al $68 \%$ women had SCH 5.6\% had subclinical hyperthyroidism $22.6 \%$ had overt hypothyroidism, $3.7 \%$ with overt hyperthyroidism. None had thyroid nodule, carcinoma and post-partum thyroiditis. ${ }^{6}$ Out of 402 cases who delivered, $6.2 \%$ had thyroid disorders, SCH being $4.8 \%$. Dhanwal et al found $14.3 \%$ women attending a tertiary public hospital in Delhi, had hypothyroidism and majority being subclinical. ${ }^{7}$ All studies including ours showed the need of universal screening for thyroid dysfunction.

In present study, the majority of patients were between 20-25 years $(44.1 \%)$ with significant percentage of subclinical hypothyroidism. Overt hypothyroidism was more in $>35$ years and other type of thyroid dysfunction were more below 20 yrs. In a study by Nambiar et al the mean age at presentation of all types of thyroid dysfunction was $25.19+4.17$ and overt hypothyroid women had higher maternal age as compared to women in the other groups. ${ }^{2}$ This study gives a similar result like our study may be because of the current trend of older women becoming pregnant. Majority of the women were primigravida $(58.4 \%)$. Significant number of primi were there in each group. Shah JM et al in a prospective study observed, $45 \%$ women primigravida and $55 \%$ multigravida. ${ }^{8}$ In a study by Dave A et al $42 \%$ had history of previous thyroid disease. ${ }^{9}$ In present study group $10.9 \%$ cases had previous history of thyroid disease. Spontaneous abortion was the most common 
complication found in previous pregnancy. Caesarean section for meconium stained amniotic fluid was second common complication, $2.4 \%$.

Since we do first trimester screening most of the disease were diagnosed in the first trimester, mostly after 8 weeks, only $0.4 \%$ left undiagnosed. In a cross sectional study by Rajput $\mathrm{R}$ et al median gestational age at diagnosis was 8 weeks 5 days, in first trimester itself. ${ }^{10}$ No obstetric complications were found in 224 (49.9\%) of women. Pre-eclampsia and miscarriage were the most common complications found associated with thyroid dysfunction 47 ladies each $(10.5 \%)$. Mohanthi $\mathrm{R}$ et al reported an incidence $15 \%$ of hypertension in SCH. ${ }^{11}$ Abalovich et al showed that untreated hypothyroidism, subclinical, or overt, at the time of conception is associated with miscarriage rate of $31.4 \%$ compared with $4 \%$ in euthyroid subjects at conception. ${ }^{12}$ Debanjali Sarkar in a study found that minimal degree of hypothyroidism can increase the rate of miscarriage and fetal death and can be an underlying etiology of recurrent pregnancy loss. ${ }^{13}$ Prolonged period of infertility, recurrent abortions, abruption placenta, PPROM, preterm labor, post-partum hemorrhage were found significantly high in overt hypothyroidism than others. Normocytic anemia was found significantly high in subclinical hypothyroidism $(9.8 \%)$ Abdominal delivery was high in overt hypothyroidism.

Majority of fetus had no complications. Fetal growth restriction, presence of meconium stained amniotic fluid and fetal distress, were the complications met with in this study. Complications like miscarriage, growth restriction, preterm labor, meconium stained amniotic fluid, and increased perinatal mortality were found in a study by $\mathrm{S}$ $\mathrm{O}$ Le Beau et al. ${ }^{14}$ Present study showed significant percentage of maternal complications associated with thyroid disorder in spite of early detection and treatment Veronica A et al emphasizes the importance of detection of significant thyroid dysfunction at time of conception or as early as possible in first trimester and timely treatment can optimize maternal and fetal outcome. ${ }^{15}$

\section{CONCLUSION}

Study concluded that thyroid dysfunction in pregnancy is associated with various types of maternal complication. SCH was present in $72.2 \%$ ladies, overt hypothyroidism in $20.7 \%$, Sub clinical hyperthyroidism for $1.3 \%$ women, overt hyperthyroidism for $5.3 \%$, thyroid nodule and thyroid cancer for $0.4 \%$ cases. Significant percentage of SCH was present between 20-25 yrs. Overt hypothyroidism was significantly high in women $>35$ Yrs and other type of thyroid dysfunction were significantly more below 20yrs. No difference in socio-economic class and place in each type of thyroid dysfunction. Significant numbers of primigravida were there in each group. In overt hypothyroidism, abdominal delivery was significantly high.
Preeclampsia and spontaneous miscarriages were the most common complication found associated with thyroid dysfunction, anemia and recurrent pregnancy loss follows. Prolonged period of infertility, recurrent pregnancy loss, post-partum hemorrhage and abruption were found significantly high in overt hypothyroidism than others. Anemia was found significantly high in subclinical hypothyroidism. Need of Caesarean section for meconium stained amniotic fluid premature rupture of membrane, fetal distress, oligohydramnios was noted significantly. Overt hypothyroidism being the major contributor.

Thyroid dysfunction is a condition that should be treated aggressively and affects a significant number of women during pregnancy associated with adverse maternal outcome. Thyroid dysfunction is arising in India with recent studies highlighting tremendous increase in incidence. The result of the present study indicates that thyroid dysfunction has got many adverse effects on pregnancy especially if not detected and treated timely. . Hence it is important to screen all pregnant women in the first antenatal visit itself in order to facilitate early diagnosis. They should be properly counseled regarding the adverse outcome of the disease.

\section{ACKNOWLEDGMENTS}

Authors would like to acknowledge the faculties and other staff members of the Dept of OBG Government TD Medical College Alappuzha in their respective assistance in preparing this manuscript.

Funding: No funding sources Conflict of interest: None declared

Ethical approval: The study was approved by the Institutional Ethics Committee

\section{REFERENCES}

1. Sahu MT, Das V, Mittal S, Agarwal A, Sahu M. Overt and subclinical Thyroid dysfunction among Indian pregnant women and its effect on maternal and fetal outcome. Arch Gynecol Obstet. 2010;281(2)215-20.

2. Nambiar V, Jagtap VS, Sarathi V, Lila AR, Kamalanathan S, Bandgar TR et al. Prevalence and impact of thyroid disorders on maternal outcome in Asian-Indian pregnant women. J Thyroid Res. 2011 Jul 17;2011.

3. Stagnaro-Green A, Abalovich M, Alexander E, Azizi F, Mestman J, Negro R et al. Guidelines of the American Tyroid Association for the diagnosis and management of thyroid disease during pregnancy and postpartum. Thyroid. 2001;21(10):1081-125.

4. Chan S, Franklyn J. Thyroid hormone in pregnancy and fetus. Progress in Obstet Gynecol. 75-93.

5. David James. High risk pregnancy management options; $4^{\text {th }}$ Ed. Chapter $45 ; 813-86$. 
6. Ajmani SN, Aggarwal D, Bhatia P, Sharma M, Sarabhai V, Paul M. Prevalence of overt and subclinical thyroid dysfunction among pregnant women and its effect on maternal and fetal outcome. J Obstet Gynecol India. 2014;64(2):105-10.

7. Dhanwal DK, Prasad S, Agarwal AK, Dixit V, Banerjee AK. High prevalence of subclinical hypothyroidism during first trimester of pregnancynin North India. Indian $J$ Endocrinol Metab. 2013;17:2814.

8. Shah JM, Mehta MN, Viradia HB. Screening for thyroid dysfunction during pregnancy. Thyroid Res Prac. 2013;10(2):65.

9. Dave A, Maru L, Tripathi M. Importance of universal screening for thyroid disorders in first trimester of pregnancy. Indian J Endocrinol Metab. 2014;18(5):733-6.

10. Rajput R, Goel V, Nanda S, Rajput M, Seth S. Prevalence of thyroid dysfunction among women during the first trimester of pregnancy at a tertiary care hospital in Haryana; Indian J Endocrinol Metab. 2015; 19:416-8.
11. Mohanty R, Patnaik S, Ramani B. Subclinical Hypothyroidism during pregnancy: A clinical review. Indian J Clini Prac. 2014;25(5):249-254.

12. Abalocich. Thyroid dysfunction during and after pregnancy. J Clin Endocrinol Metab. 2007;929(8):S1-47.

13. Sarkar D. Recurrent pregnancy loss in patients with thyroid dysfunction. Indian $\mathrm{J}$ Endocrinol Metab. 2012;16(2):S350-1.

14. LeBeau SO, Mandel SJ. Thyroid disorders during pregnancy. Endocrinol Metab Clin North America. 2006;35(1):117-36.

15. Learoyd DL, Preda VA. Recognising and managing thyroid dysfunction in pregnancy. Medicine Today 2014;15(12):47-54.

Cite this article as: Manju VK, Sathiamma PK. Maternal outcome in thyroid dysfunction. Int $\mathbf{J}$ Reprod Contracept Obstet Gynecol 2017;6:2361-5. 